Planetary tides on the Sun influence solar activity, which in turn causes an increase in the solar cosmic ray flux reaching the Earth's upper atmosphere. This triggers unusual movements of large air masses, thus effecting the Earth's rate of rotation and subsequently triggering earthquakes. This, in a nutshell, is known as the 'Jupiter effect' mainly because Jupiter exerts the largest tidal influence on the Sun (this influence is proportional to $M d^{-3}$ where $M$ is the planetary mass and $d$ its distance from the Sun, so that Mercury, Venus, Earth, Mars, Jupiter, Saturn, Uranus, Neptune and Pluto have relative tidal effects on the Sun in the ratios of $4.1: 97.3: 44.2: 1.4: 100: 49: 0.09$ : $0.03: 0.001$ ) assuming the Jovian effect to be 100.) It was first proposed by K. D. Wood (Nature, 240, 91; 1972) and popularised in The Jupiter Effect by John Gribbin and Stephen Plagemann (Macmillan London; Walker, New York; 1974) which generated considerable discussion and also a degree of criticism, counter argument and rejoinder (Icarus, 26, 257, 268, 270 ; 1975).

Jean Meeus, the most severe critic, pointed out that according to this interpretation of the data, planetary alignments have an undetectable effect on sunspot activity In his opinion the effect doesn't exist. But Gribbin and Plagemann's argument is that planetary alignments are important and none more so than the 1982 superconjunction when Jupiter, Saturn, Uranus and Neptune will be colinear. These superconjunctions occur about every $178.73 \mathrm{yr}$. In this

cathartic activity promoting withingroup harmony although, as Durham points out, it seems a rather extravagant method of promoting peace within the village. Instead, he suggests that the tradition is directly related to competition for a scarce resouce: game animals. The Mundurucu rely on game for high quality protein, and this is reflected in the importance of game in their religious rituals. Head hunting rituals also involved reference to game. A successful warrior was referred to as 'mother of the Peccary', referring to the fact that other village members were viewed as equivalent to game, and that obtaining a head trophy magically increased the fertility of game animals. This fits in well with Durham's interpretation that intergroup warfare is a form of individually selfish competition for game. More puzzling from the point of view of individual benefits, is the tradition that a successful warrior abstains from sexual intercourse for three rainy seasons. Not much good for individual fitness!

\section{Planetary alignments don't cause earthquakes}

\author{
from David W. Hughes
}

time Jupiter travels 15 times round its orbit plus an extra $24^{\circ}$, Saturn performs six revolutions plus $24^{\circ}$, Uranus two revolutions plus $46^{\circ}$ and Neptune one revolution plus $30^{\circ}$ Meeus points out that a period of 178.73 yr should have no special significance as Saturn, Uranus and Neptune exert a rather minor tidal influence on the Sun in comparison with, say, Venus and Earth (see above). Tides raised on the Sun by Venus, Earth and Jupiter have a period of four months and this should be the dominant factor if any interrelation exists. The temporal similarity between the 11.1-yr cycle of solar activity and the 11.86 -yr orbital period of Jupiter is thought to be entirely fortuitous. Also there are two tidal bulges produced on the Sun, one at the sub-planetary point and the other diametrically opposite, so the planets don't have to be all on the same side of the Sun as was asserted by Gribbin and Plagemann. The heliocentric opposition of January 16, 1901 should have been just as effective as the forthcoming superconjunction of 1982 .

Despite these arguments Gribbin and Plagemann still "see no reason yet to discard the suggestion that planetary alignments do affect sun-

Durham argues that the very scarcity of protein which precipitated the warfare would make procreation at that moment an unprofitable business (he doesn't say whether the whole tribe abstains), and that the warrior's payoff comes in other ways, such as exemption from future raids.

Durham's approach to interpreting cultural traditions of warfare is entertaining and makes intuitive common sense to a biologist, but whether it will really provide any predictive insight into differences between cultures rather than merely explaining after the fact, remains to be seen.

Returning to the costs and benefits of aggressive competition for food in animals, Carpenter and MacMillen, (Science, 194, 639; 1976) have recently reported a quantitative study of territoriality in the Hawaiian Honeycreeper Vestiaria coccinea. Individuals of this species sometimes defend a feeding territory on flowering trees of the genus Metrosideras. Carpenter and MacMillen argue that territorial aggression spots, let alone the more fundamental contention that solar activity affects the Earth's rotation and therefore earthquakes."

One way to solve this disagreement is to look at earthquake data to see whether the occurrence of earthquakes has a periodicity of about 179 yr. Gribbin and Plagemann peer into the future, predicting that 1982 is going to be a year of considerable activity and a possible date for the unwelcome movement of the San Andreas fault in California.

Also addressing this problem W.-H. Ip of the Department of Applied Physics and Information Science, University of California, San Diego, does without the crystal ball and takes a more orthodox scientific approach. In a recent issue of Icarus $(29,435 ; 1976)$ he describes how Yu Shen (in Ke Yue Shi Yan (Scientific Experiments), May 1975 page 22) has divided the period between $1000 \mathrm{AD}$ and the present into quiet and disturbed periods of earthquake activity using ancient records of earthquakes in Northern China. Ip finds that since $780 \mathrm{BC}$ only one of the 125 recorded earthquakes with intensities corresponding to 6 on the Richter scale coincided with a heliocentric planetary alignment, this one occurring in $1624 \mathrm{AD}$. Since $1000 \mathrm{AD}$ none of the eleven earthquakes with intensities of 8 has coincided. These facts really do speak out against the Jupiter effect and Californians can, one hopes, rest more easily in their beds; San Andreas will undoubtedly move some time in the future but 1982 is no more likely to be the year than any other.

will be economically advantageous as a means of securing food only if the gain in terms of increased nectar yield through excluding competitors exceeds the energy cost of territorial defence. Thus, for a given efficiency of excluding nectar thieves, territoriality will pay only if the average nectar productivity is above a certain level. (If productivity is low, no amount of exclusion of rivals will pay for itself.) At very high levels of productivity, there is so much nectar available that exclusion of rivals is not necessary, so honeycreepers should only defend food territories at intermediate levels of nectar production. By calculating the energy cost of maintenance for a territorial and nonterritorial bird, as well as estimating the nectar production in each territory and the efficiency of exclusion of rivals, Carpenter and MacMillen could predict in a quantitative manner which birds should show territorial aggression, and which individuals should not. For nine out of ten birds (six territorial and four non-territorial) the behaviour was 